\title{
ORIGINAL ARTICLE \\ Genetic and ecological data reveal species boundaries between viviparous and oviparous lizard lineages
}

\author{
L Cornetti $^{1,2,6}$, GF Ficetola ${ }^{3,4}, \mathrm{~S}$ Hoban $^{5}$ and C Vernesi ${ }^{1}$
}

Identification of cryptic species is an essential aim for conservation biologists to avoid premature extinctions of 'unrecognized' species. Integrating different types of data can undoubtedly aid in resolving the issue of species delimitation. We studied here two lineages of the common lizard Zootoca vivipara that display different reproductive mode (the viviparous $Z$. v. vivipara and the oviparous Z. v. carniolica) and that overlap their distributional ranges in the European Alps. With the purpose of delimiting species' boundaries, we analyzed their ecological, genetic and natural history features. More than 300 samples were collected and analyzed at cytochrome $b$ and 11 microsatellites loci for investigating genetic variation, population structure, individual relatedness and evolutionary histories of the two lineages. Additionally, we compared their ecological niches using eight ecological variables. Genetic data showed contrasting patterns of genetic structure between the two lineages, different demographic dynamics and no hybridization events. Also strong ecological differences (such as temperature) emerged between the two lineages, and niche overlap was limited. Taken together, these results indicate that Z. v. vivipara and Z. v. carniolica should be recognized as two separate species, and particular conservation consideration should be given to the oviparous lineage that tends to live in areas threatened by increasing impact of human activities. However, recent and rapid climate warming might determine an increasing risk for the persistence of the viviparous lineage, being adapted to cold environments.

Heredity (2015) 115, 517-526; doi:10.1038/hdy.2015.54; published online 1 July 2015

\section{INTRODUCTION}

Accurate species delimitation is a basic objective in evolutionary systematics for understanding extant levels of biodiversity and adopting effective conservation strategies. Identifying cryptic species is recognized as fundamental to avoid premature extinctions of 'unrecognized' species, in particular in the light of current ongoing global changes (Koh et al., 2004). It has been widely recognized that using only morphological traits for taxonomic inference tends to underestimate cryptic biodiversity, while genetic and phylogenetic tools contribute greater resolution and accuracy for taxonomy and systematics (Bickford et al., 2007). However, in some cases, using only phylogenetic methods can give inconclusive results. To resolve species delimitation fully, a multidisciplinary approach, defined 'integrative taxonomy', has emerged with the aim of combining many different types of data (for example, phylogenetics, morphology, behavior, population genetics, ecology; Dayrat, 2005).

The integrative taxonomy approach has been recently successful for many species groups, by combining morphological data with additional molecular phylogenies (for example, Singhal and Moritz, 2013), behavioral information (for example, Bourguignon et al., 2013) or ecological data (for example, Raxworthy et al., 2007). For instance, closely related species may show adaptations that allow them to exploit distinct environments, therefore showing differences for their ecological niche, although detecting these differences may be challenging. Only recently robust analytical tools have been developed for an objective assessment of niche differences (Broennimann et al., 2012). In this study, we analyze species delimitation in the lacertid genus Zootoca using an integrative approach.

The lizard Zootoca vivipara is among the reptiles with the widest distribution range and northernmost distribution limits for a lizard (Surget-Groba et al., 2001). However, this widespread lizard has strong intraspecific structure, with striking different reproductive mode among lineages. Specifically Z. v. vivipara, corresponding to mitochondrial clade E according to Surget-Groba et al. (2006), and occupying the great majority of the European distributional range of the species, is a viviparous lineage: embryos develop inside eggs within the body of the mother followed by birth to live offspring. Meanwhile two allopatric oviparous lineages, namely Z. v. louislantzi (Arribas, 2009) and Z. v. carniolica (Mayer et al., 2000), live in the Pyrenees and in the Central Eastern Alps, respectively. No morphological traits clearly distinguish any Z. vivipara lineages (Guillaume et al., 2006), while mitochondrial and nuclear sequences data clearly resolved phylogenetic relationship, with Z. v. carniolica being the sister lineage to all the others. Although distributional ranges of $Z$. v. vivipara and Z. v. louislantzi do not overlap in Western Europe, Z. v. vivipara and Z. v. carniolica are parapatric in the European Alpine chain. This pattern was likely generated by quaternary temperature oscillations

${ }^{1}$ Department of Biodiversity and Molecular Ecology, Research and Innovation Centre, Fondazione Edmund Mach, Via E. Mach 1, San Michele all'Adige (TN), Italy; ${ }^{2}$ Dipartimento di Scienze della Vita e Biotecnologie, University of Ferrara, via Borsari 46, Ferrara, Italy; ${ }^{3}$ Laboratoire d'Ecologie Alpine (LECA), Université Grenoble-Alpes, Grenoble, France; ${ }^{4}$ LECA, CNRS, Grenoble, France and ${ }^{5}$ National Institute for Mathematical and Biological Synthesis, Knoxville, TN, USA

Correspondence: Dr L Cornetti, Institute of Evolutionary Biology and Environmental Studies, University of Zurich, Wintherthurerstrasse 190, Zürich 8057, Switzerland.

E-mail: lucacornetti@gmail.com

6Present address: Institute of Evolutionary Biology and Environmental Studies, University of Zurich, Wintherthurerstrasse 190,8057 Zürich, Switzerland.

Received 20 February 2015; revised 18 May 2015; accepted 18 May 2015; published online 1 July 2015 


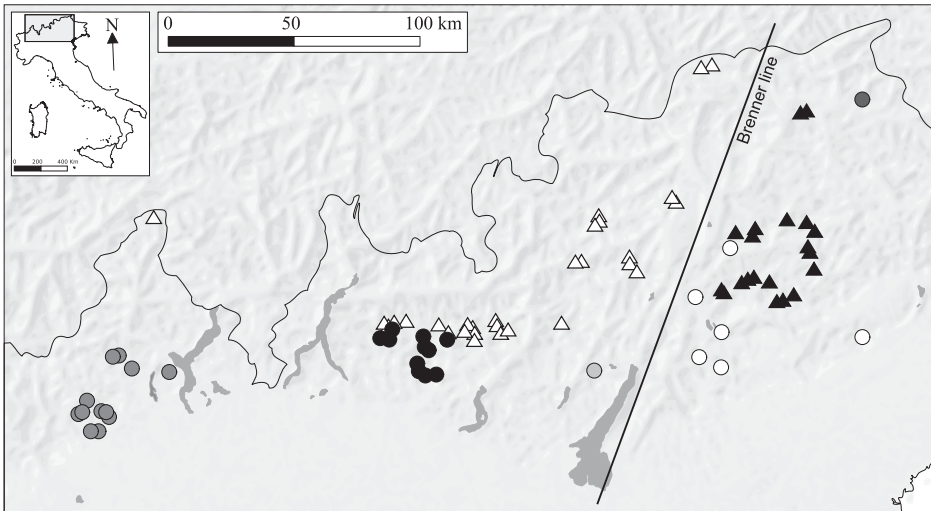

\begin{tabular}{|l|}
\hline Z. v. vivipara \\
$\Delta$ West of Brenner line \\
A East of Brenner line \\
Z. v. camiolica \\
- Rasun \\
- Eastern Trentino \\
- Lombardy \\
- Ampola \\
- Piedmont
\end{tabular}

Figure 1 Sampling sites of common lizard across the Italian Alps. Triangles and circles represent locations where Z. v. vivipara and Z. v. carniolica were collected, respectively (according to mtDNA results). Different gray scales indicate the homogeneous genetic groups identified by STRUCTURE analyses and describe the two and five clusters inferred for Z. v. vivipara and Z. v. carniolica, respectively. Lakes are shown in dark gray.

that strongly influenced the demographic history of the species, likely promoting the evolution of viviparity (Surget-Groba et al., 2006)

In reptiles, viviparity often evolves as an adaptation to cold climates (Pyron and Burbrink, 2014). Pleistocene climatic changes forced presumably still oviparous $Z$. vivipara populations to disperse to suitable southern areas of Europe. Pleistocene glacial movements have acted as a strong selective pressure in some populations between the Balkan Peninsula and Southern Russia, where viviparity likely arose, and probably facilitated the recolonization of the entire continent during interglacial periods (Surget-Groba et al., 2001). Conversely, surviving populations in isolated Italian refugia (currently classified as Z. v. carniolica), remained oviparous and well adapted to warmer temperatures (Surget-Groba et al., 2002). Recolonization pathways brought the two allopatrically differentiated lineages into secondary contact in the Alps. In the southern margin of species distribution the two lineages tend to live in moist mountainous habitat with $Z$. $v$. carniolica also present in a few relict spots in the Po plain (SurgetGroba et al., 2002). In fact, oviparous and viviparous lineages are presumed to be better adapted to warmer and colder environmental conditions, respectively, and a differential altitudinal distribution has been reported, with the viviparous lineage tending to live at higher altitudes (Cornetti et al., 2014). However, the two lineages show a definite overlap of their altitudinal distributions in the Alps, as confirmed by recent findings of syntopy areas (Lindtke et al., 2010; Cornetti et al., 2015), and quantitative analyses of niche differentiation between them are lacking.

This system provides the opportunity for combining molecular, life history and ecological data to assess species delimitation between $Z$. v. vivipara and $Z$. v. carniolica. Elucidating the taxonomic status of the carniolica lineage has major conservation implications. Overall, $Z$. vivipara is a widespread, least concern species (IUCN, 2014). However, local extinctions and population declines have been reported for Z. v. carniolica; during the past century, many low-altitude populations have disappeared, probably because of the high anthropization level in lowlands and/or because of climate changes (Sindaco et al., 2006). Clear delimitation between the two lineages would therefore imply that $Z$. v. carniolica is a taxon of conservation concern. Furthermore, if the two species show strong ecological differences, management strategies should be definitively different among them.

In this study we apply an integrative approach combining mitochondrial phylogeny, population genetic and life history analyses using autosomal microsatellites, and ecological niche data for providing an accurate image of species boundaries.

\section{MATERIALS AND METHODS}

\section{Sample collection and molecular techniques}

Lizard samples were collected across the Alpine chain with the objective to cover most of the overlapping distribution of Z. v. carniolica and Z. v. vivipara (Figure 1 and Supplementary Table S1). Since their distributions are strictly correlated with moist habitat, our sampling primarily focussed on locations with this kind of habitat. Some of the intervening, unexplored zones might be suitable habitat, and we cannot exclude that the two subspecies can be found in other locations. Lizards were captured by hand, collecting $\sim 0.5 \mathrm{~cm}$ of tail tissue and immediately released. Individual GPS coordinates of each sample and altitude were recorded. Tissue samples were stored in 95\% ethanol until DNA extraction. The sample set $(N=304)$ used in the present study was obtained by merging tissue collections (191) used in Cornetti et al., (2014) and newly collected samples (113, summer 2012, thus far unpublished). Genomic DNA was extracted using the DNeasy Blood and Tissue Kit (QIAGEN Inc, Hilden, Germany) following manufacturer's instructions. The newly collected samples were amplified and sequenced for a 385 base pair fragment of mtDNA cytochrome b (cytb) according to Cornetti et al. (2014) for lineage identification. In addition, and for the first time on the entire sample set, 11 nuclear microsatellites (Lv-3-19, Lv-4-72, Lv-4-alpha, Lv-2-145, Lv-4-X, Lv-4-115 and Lv-1-139 from Boudjemadi et al. (1999); B107, B114, C103, D109 from Remon et al. (2008)) were amplified in four multiplexes according to the thermocycling conditions specified in Supplementary Table S2. This study is distinct from two other recent works in this system. Cornetti et al. (2014), used sequences of mitochondrial and nuclear genes to examine Z. v. carniolica using a phylogenetic approach, supported by dating the divergence between the species; no microsatellites were used. Cornetti et al. (2015) examined hybridization dynamics at very fine scale, with different samples. Here, we compare the ecological and genetic patterns (based on microsatellites marker for the first time on the entire data set) at broad scale in the two lineages where they overlap their distributional ranges (European Alps). None of the sample analyzed in Cornetti et al. (2015) is included in the present study. Although we here use some of the samples of Cornetti et al. (2014), we have additional samples, and genotyped all samples at nuclear microsatellites specifically for this work.

\section{Data analysis: mtDNA and microsatellites}

Sequences were successfully obtained for all samples and aligned with deposited haplotypes (AY714882-AY714929) coming from Alpine area. This data set was used to build a median joining network using Network 4.612 (Bandelt et al., 1999 ) in order to discriminate between the two Z. vivipara lineages since no morphological traits clearly distinguish them (Guillaume et al., 2006). Cytb variability was assessed using ARLEQUIN 3.5.1.2 (Excoffier and Lischer, 2010) by analyzing nucleotide diversity, mean number of pairwise differences (intralineages) and $F_{\text {st }}$ comparison (between lineages).

The 11 microsatellites were first examined for presence of null alleles, allele dropout and scoring errors using MicroChecker (Van Oosterhout et al., 2004). 
ARLEQUIN (Excoffier and Lischer, 2010) was used to calculate number of alleles $\left(N_{\mathrm{a}}\right)$, expected $\left(H_{\mathrm{e}}\right)$ and observed $\left(H_{\mathrm{o}}\right)$ heterozygosity, deviation from Hardy-Weinberg equilibrium and linkage disequilibrium between pairs of loci, while allelic richness $\left(A_{\mathrm{r}}\right)$ was calculated with Fstat (Goudet, 1995). Before intralineage analysis, we used Factorial correspondence analysis implemented in Genetix (Belkhir et al., 2004) to exclude any possible discrepancy between mitochondrial and nuclear assignment, classifying each individual as $Z$. $v$. vivipara or Z. v. carniolica according to mtDNA determination.

For detecting possible hybridization events, we used the Bayesian algorithms of NewHybrids 1.1 (Anderson and Thompson, 2002) on the entire data set $(N=304)$. NewHybrids calculates, for each individual posterior probability of assignment in different categories: pure parental populations, F1 hybrids, F2 hybrids or backcrosses. We used using default settings and tried both uniform and Jeffreys-like priors. We ran 500000 iterations after a burn-in of 100000 .

The most likely number of genetic clusters $(K)$ in our sample set was evaluated using a hierarchical analysis with STRUCTURE 2.3.2 (Pritchard et al., 2000). We initially ran 10 replicates consisting of 1000000 iterations after a burn-in of 250000 on the entire data set $(N=304)$ with $K$ ranging from 1 to 10. After the identification of two clearly distinct genetic clusters (see Results section) corresponding to the two $Z$. vivipara lineages, STRUCTURE was also used to investigate population structure within $Z$. v. carniolica $(N=114)$ and $Z$. v. vivipara $(N=190)$ with the same setting as above. The most likely number $K$ that best explained genetic structure in the entire data set and within lineages, was estimated using STRUCTURE HARVESTER (Dent and Von Holdt, 2012), analyzing both $L(K)$ (Pritchard et al., 2000) and $\Delta K$ (Evanno et al., 2005). Additional analyses, for investigating intralineage genetic structure and presence of barriers to gene flow, were performed in BARRIER 2.2 (Manni et al., 2004, see Supplementary Methods).

Evidence of possible reduction in effective population size $\left(N_{\mathrm{e}}\right)$ was investigated using M_P_VAL (Garza and Williamson, 2001), as well as the Wilcoxon test implemented in bottleneck (Piry et al., 1999). Both methods contemplate a complex mutational model, that microsatellites markers usually exhibit (Di Rienzo et al., 1994; Peery et al., 2012); we ran M_P_VAL setting the mean size of multistep mutation and the proportion of multistep events to 3.1 and 0.22 , respectively, as suggested by Peery et al. (2012). In bottleneck, we used a two-phase mutational model (with $78 \%$ stepwise mutation model and $22 \%$ infinite allele model) and a variance among multiple steps equal to 12 (Piry et al., 1999). M-ratio is expected to decrease in shrinking populations, indicating a bottleneck in the distant past, if its value is $<0.68$ (Garza and Williamson, 2001). We explored a wide range of possible pre-bottleneck effective population sizes performing the analyses using the following $\theta$ values: $1,2,5$, corresponding to 500,1000 and 2500 individuals (using the common estimate of mutation rate of $5.0 \times 10^{-4}$ mutations/generation/locus; Estoup and Angers, 1998). The statistical significance of M-ratio was obtained after 10000 simulations of a population at equilibrium. The Wilcoxon test, in contrast, determines if a population suffered a recent reduction in population size $(0.2-$ $4 \mathrm{xN}_{\mathrm{e}}$ generation before present; Luikart and Cornuet, 1998) by evaluating the transient heterozygote excess in bottlenecked populations.

\section{Isolation by distance}

The correlation between genetic similarity and geographic distance was examined for the two lineages. Specifically, we constructed spatial autocorrelograms, a way of visualizing decay of relatedness between individuals over distance (isolation by distance). With SPAGeDi (Hardy and Vekemans 2002) we estimated the kinship coefficient derived by Loiselle et al., (1995) for all pairs of individuals within distance classes chosen so that they composed a similar number of comparisons. The purpose of this analysis was to observe whether the two species had similar degrees of relatedness among pairs of individuals on a landscape. We also created autocorrelograms with other different measures of kinship and relatedness and obtained similar results.

\section{Ecological niche differences}

We considered eight environmental variables to compare the Grinnelian niche of the two lineages. Three variables describing the thermal environment (average, minimum and maximum annual temperature) and three variables representing water availability (annual, winter and summer precipitation, potential evapotraspiration) were obtained from WorldClim (Hijmans et al., 2005). We also considered potential evapotranspiration, a climatic parameter representing the amount of atmospheric energy that is strongly related to reptile distribution (Rodriguez et al., 2005), and the normalized difference vegetation index, a proxy of primary productivity, obtained from time-series maps of the VEGETATION program (http://www.vgt.vito.be).

We used two approaches to compare the niches of the two lineages. First, we used the principal component analysis (PCA)-env approach to perform multivariate comparisons of niche overlap (Broennimann et al., 2012). PCAenv measures niche overlap on the basis of occurrence and environmental data, and has shown to be the one of the most reliable techniques for niche comparison (Broennimann et al., 2012). This method performs a PCA on the environmental spaces that is available to the two lineages. PCA-env uses a kernel density function to compute the density of occurrences in the multivariate PCA space, in order to take into account potential bias caused by unequal sampling effort. Niche overlap and niche similarity are calculated using the Schoener's D metric (Warren et al., 2008). Schoener's D ranges between 0 (lack of overlap) and 1 (complete overlap), and is particularly suitable to analyze overlap in Grinellean niches (Warren et al., 2008). The significance of niche differences/similarity between lineages was tested by comparing the observed $D$-values to null distributions, using equivalency and similarity tests (Warren et al., 2008; Broennimann et al., 2012). The equivalency tests assess whether niches of two species are equivalent, by evaluating if overlap is constant when randomly reallocating occurrences of both lineages among the two ranges. The similarity test evaluates if the niche occupied by one lineage is more similar to the niche of the other species than expected by chance, by testing if the observed overlap between the niches of the two lineages is different from the overlap between the niche of one lineage and the 'niches' of locations selected randomly from the range of the other lineage (Warren et al., 2008; Broennimann et al., 2012). Significance of tests was assessed through 999 random permutations. This analysis was repeated at both fine and broad spatial scale. At fine scale, the study area was the same analyzed for genetic analyses (that is, Italy, north of $45^{\circ} 30^{\prime} \mathrm{N}$ ), and we considered all the Z. v. vivipara and Z. v. carniolica localities available for Northern Italy (see Figure 1) for which many high-resolution data were available. For this analysis, all variables were obtained at the resolution of 30 arc-seconds, that is, $\sim 650 \times 920 \mathrm{~m}$ within the study area. To confirm that the fine-scale results correspond to the situation occurring over the whole range of the taxa, the analysis was repeated at broad spatial scale, using the whole Palearctic realm as a study area. For this analysis, we included $Z$. v. carniolica and $Z$. v. vivipara records covering the whole range of the two subspecies (Surget-Groba et al., 2002; Surget-Groba et al., 2006; Lindtke et al., 2010; Supplementary Figure S1). The spatial accuracy of most of literature records was limited, as only locality names were available, therefore this analysis was performed at the $0.1^{\circ}$ resolution $(\sim 8 \times 11 \mathrm{~km})$

To better depict differences for individual environmental variables (that is, for which variables there are differences between the two lineages), after the PCA-env analyses we used density profiles to compare the niche along the eight environmental variables (for example, Rodder, 2013). Comparisons were performed using the sm.density.compare function of the $s m$ package in $\mathrm{R}$ (Bowman and Azzalini, 2010); significance was tested using 10000 bootstraps.

\section{RESULTS}

\section{MtDNA}

Cytb analysis suggested that across the Italian Alps 114 samples were ascribable to Z. v. carniolica and 190 samples to Z. v. vivipara (Figure 2a). In Z. v. carniolica, we found 12 different haplotypes, three of which have never been observed before (KM522844KM522846), while in the Z. v. vivipara samples there were six haplotypes, one of which is novel (KM522847). Z. v. carniolica, the oviparous lineage, proved to be about one order of magnitude more variable at mtDNA than the viviparous lineage (nucleotide diversity: $0.011 \pm 0.006$ compared with $0.001 \pm 0.001$; mean number of pairwise differences: $4.26 \pm 2.12$ compared with $0.59 \pm 0.47$ ). 
a

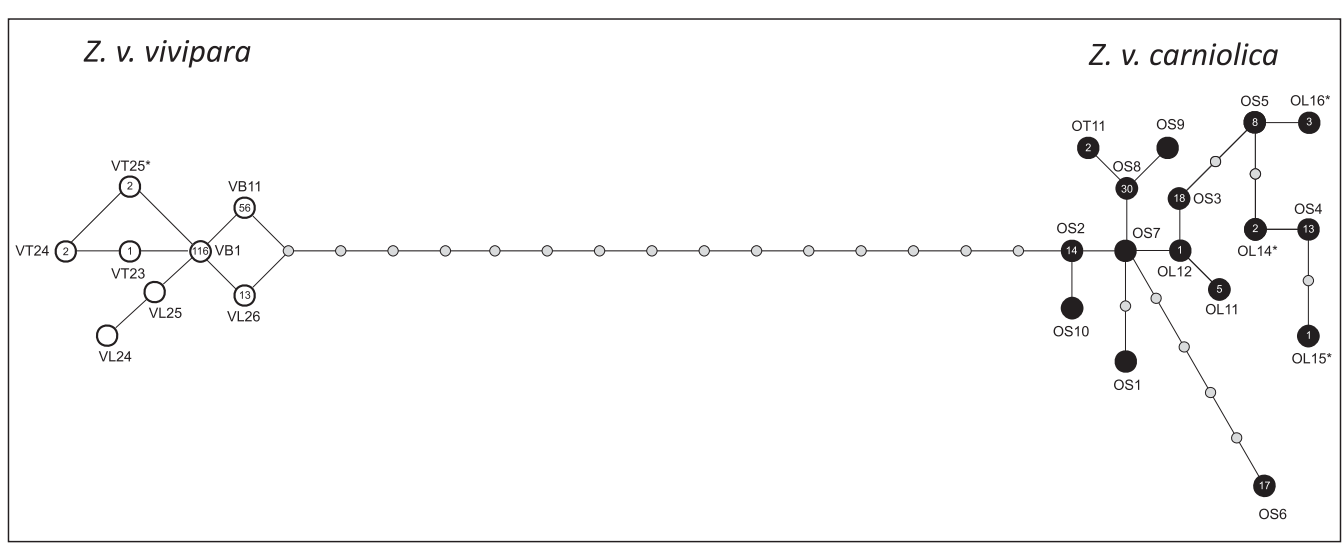

b

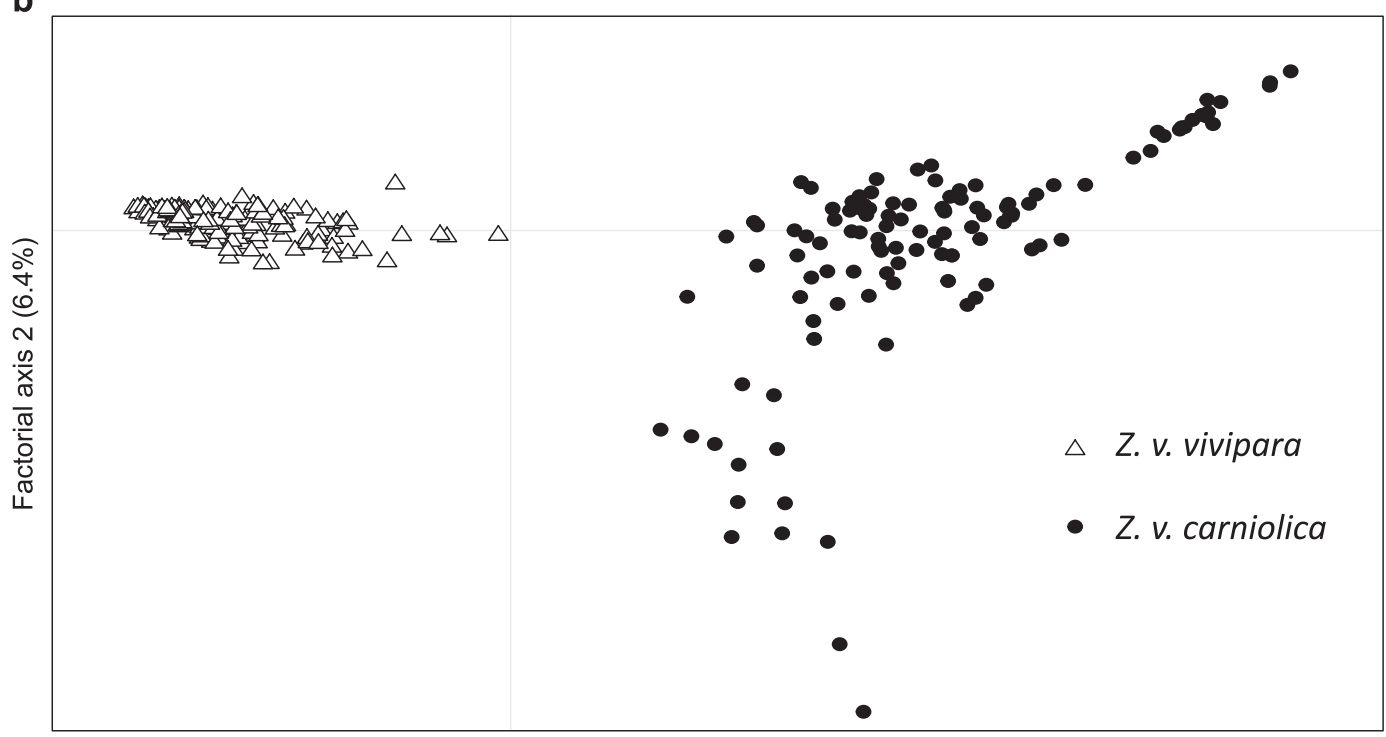

Factorial axis $1(15.8 \%)$

c

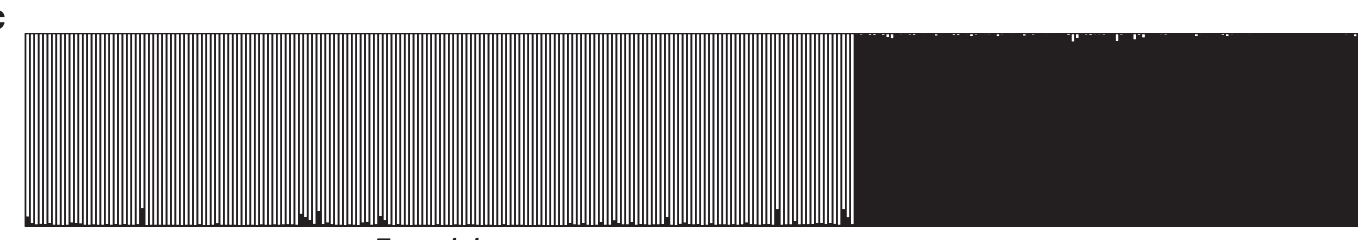

Z. v. vivipara

Z. v. carniolica

Figure 2 Median joining network of cytb haplotypes found in the Alps; Z. v. vivipara and Z. v. carniolica haplotypes are colored in white and black, respectively; number inside the circles represent the frequency, while asterisks mark sequences discovered in this study for the first time (a); factorial correspondence analysis based on six microsatellite loci discriminating two distinct groups defined according to mtDNA haplotypes (b); STRUCTURE plot describing the individual ancestry proportion of belonging to one out of the two lineages (c).

\section{Microsatellites}

All 11 nuclear microsatellite loci were polymorphic. Because of null alleles, according to MICROCHECKER results, locus Lv-1-139 was excluded from further analysis; no significant presence of null alleles, allele dropout and scoring errors were found for the remaining ten loci. In addition, no deviation from Hardy-Weinberg equilibrium and linkage disequilibrium between pairs of loci were detected. Likely because of the high genetic divergence between the two lineages, some markers could not be analyzed simultaneously in the whole data set. More specifically we could not obtain reliable fragment lengths for the loci C103 and D109 in carniolica samples. Moreover, after repeatedly finding private microvariants (incomplete repeats for given alleles) in carniolica samples for loci Lv-3-19 and Lv-4-7, we sequenced both fragments in some homozygous individuals and found a recurring deletion in the flanking region of these loci. When all 304 samples were analyzed together, Lv-3-19 and Lv-4-72 were, therefore, treated with caution. In fact, for intraspecific comparisons, we conducted analyses on two data sets, with and without Lv-3-19 and Lv-4-72. Since we obtained similar results with both marker sets, we presented the more conservative results obtained with six microsatellites (B107, B114, Lv-4-alpha, Lv-2-145, Lv-4-X and Lv-4-115).

Factorial correspondence analysis clearly separated individuals of the two lineages (based on mtDNA) into two groups, with no overlapping samples (Figure $2 \mathrm{~b}$ ) and any evidence of contrasting 
assignment based on mtDNA and microsatellites. The high genotypic divergence was also confirmed also by the mean number of private alleles per locus, 6.3 and 7.5 in Z. v. carniolica and Z. v. vivipara, respectively and significant $F_{\text {st }}(0.1198)$ and $R_{\text {st }}(0.5782)$ values for comparison between lineages.

\section{Intraspecific genetic structure and hybridization}

STRUCTURE results revealed that the most likely number of genetic groups was two according to the $\Delta K$ approach that generally shows the uppermost clustering level (Supplementary Figure S2; Evanno et al., 2005), corresponding exactly to the two Z. vivipara lineages studied here. We then specifically tested for hybridization in our sample set running a STRUCTURE analysis fixing $\mathrm{K}=2$ : the results clearly rejected the occurrence hybrid individuals (Figure 2c) In addition, NewHybrids assigned all samples to their pure parental lineage with a probability above $99 \%$.

\section{Intra-lineage genetic structure and life history}

The pattern of genetic variation in microsatellites (indicated by scattering of individuals, see Figure 2b) appeared wider in $Z$. $v$. carniolica than in Z. v. vivipara, suggesting a more pronounced genetic structure within the oviparous lineage than in the viviparous one. However, more detailed analyses were performed for understanding genetic variation within lineages using eight (B107, B114, Lv-4-alpha, Lv-2-145, Lv-4-X, Lv-4-115, Lv-3-19 and Lv-4-72) and nine loci (B114, Lv-4-alpha, Lv-2-145, Lv-4-X, Lv-4-115, Lv-3-19, Lv-4-72, C103 and D109) for Z. v. carniolica and Z. v. vivipara, respectively (marker B107 was monomorphic in Z. v. vivipara).

The intralineage genetic structure investigated using STRUCURE revealed that for $Z$. v. carniolica, the most likely number of distinct genetic clusters was five, as both $L(K)$ and $\Delta K$ showed the highest mean posterior probability values for $K=5$ (Supplementary Figure S5). The clustering analysis clearly divided the distributional range of

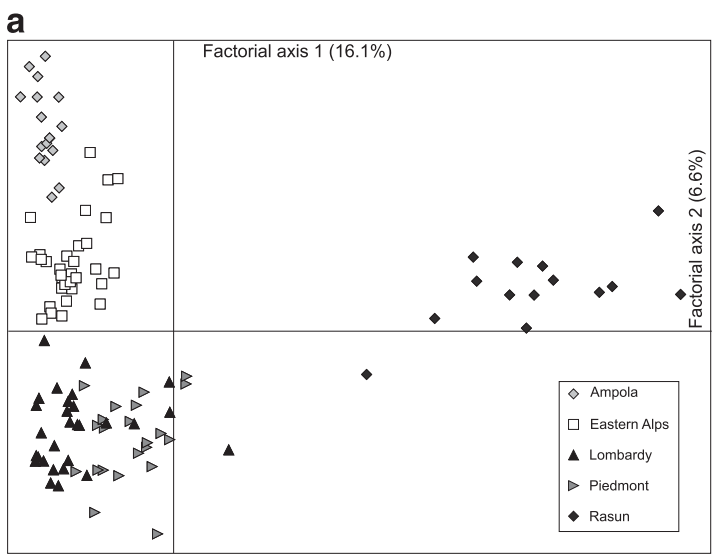

b

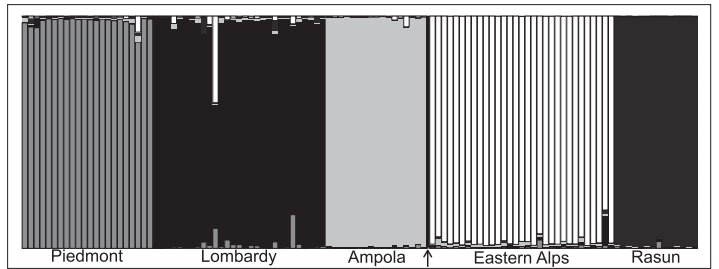

oviparous lineage in three parts: Western Alps (Piedmont); Central Alps (Lombardy); and Eastern Alps. In addition, two single populations (Ampola and Rasun) constituted distinct groups (Figure 3). All clusters displayed significant pairwise $F_{\text {st }}$ values, varying from 0.15 to 0.35 . In contrast, Z. v. vivipara lineage, showed weaker substructure, with two statistically supported clusters, according to $\Delta K$ (though the highest posterior probability for $L(K)$ was obtained for five groups $(K=5)$, mean $q$-values rarely exceeded 0.5 , meaning that inconsistent and ambiguous groups were found, Figure 3, Supplementary Figure S6, with a significant pairwise $F_{\text {st }}(0.032$, $P=0.001)$. The results obtained with STRUCTURE were consistent with BARRIER output (Supplementary Figures S7-9). Further genetic analyses were conducted within the seven groups (five in carniolica and two in vivipara) identified by STRUCTURE. The five $Z$. v. carniolica clusters included populations from 14 to 32 individuals. Number of alleles ranged from 4.4 (Ampola) to 9.5 (Piedmont); similarly, allelic richness assessed Ampola cluster as the less genetically variable. Observed heterozygosity varied from 0.47 to 0.59 , while expected heterozygosity from 0.57 to 0.77 . On the other hand, the two vivipara groups, perhaps because of higher sample size (96 and 94 samples), displayed more genetic variation (Table 1).

Four out of five clusters of Z. v. carniolica showed significant evidence of reduction in population size according to M_P_VAL results, while no evidence of bottleneck events were highlighted in $Z$. v. vivipara. No significant signal of heterozygosity excess was found using the Wilcoxon test implemented in bottleneck, meaning that neither vivipara nor carniolica clusters suffered from recent reduction in effective population size (Table 1).

\section{Isolation by distance}

The two species showed different patterns in spatial genetic structure as summarized in the autocorrelograms (Figure 4). $Z v$. carniolica displayed much higher relatedness over the first $50 \mathrm{~km}$ of distance $(>0.10)$,

C

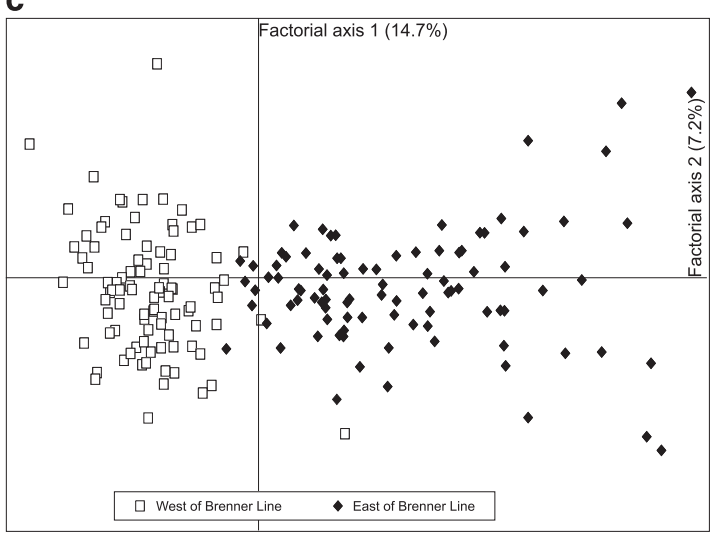

d

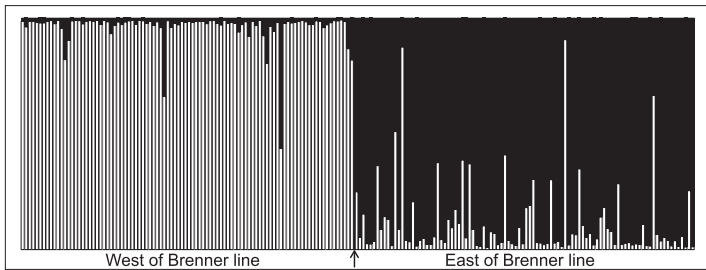

Figure 3 Factorial correspondence analysis of genotypic variation ( $\mathbf{a}$ and $\mathbf{c}$ ) and clustering analyses (b and $\mathbf{d}$ ) of Z. v. carniolica (a and $\mathbf{b}$ ) and Z. v. vivipara (c and d) lineages across the Italian Alps. In the STRUCTURE plots, the individuals are ordered by longitude (from West to East) and the 'Brenner line' is indicated by a vertical black bar and an arrow. 
Table 1 Microsatellites genetic variability and demographic inferences about $Z$. vivipara populations using M-ratio test and the Wilcoxon test of heterozygosity excess ( $\boldsymbol{P}$-value)

\begin{tabular}{|c|c|c|c|c|c|c|c|c|c|c|}
\hline Population & $N$ & $N_{a}$ & $A_{\mathrm{r}}$ & $H_{0}$ & $H_{\mathrm{e}}$ & M-ratio & $\theta=1$ & $\theta=2$ & $\theta=5$ & Wilcoxon test (bottleneck) \\
\hline Ampola & 17 & 4.4 & 3.9 & 0.51 & 0.57 & 0.59 & 0.003 & 0.011 & 0.028 & 0.464 \\
\hline Eastern Alps & 32 & 9.6 & 6.5 & 0.59 & 0.65 & 0.68 & 0.032 & 0.062 & 0.107 & 0.990 \\
\hline Piedmont & 29 & 9.5 & 7.1 & 0.59 & 0.77 & 0.76 & - & - & - & 0.527 \\
\hline Rasun & 14 & 5.5 & 5.2 & 0.47 & 0.69 & 0.43 & 0.00001 & 0.00001 & 0.00001 & 0.097 \\
\hline \multicolumn{11}{|l|}{ Z. v. vivipara } \\
\hline East of Brenner line & 96 & 14.8 & 14.5 & 0.71 & 0.79 & 0.72 & - & - & - & 0.632 \\
\hline West of Brenner line & 94 & 11.2 & 11.0 & 0.63 & 0.73 & 0.70 & - & - & - & 0.367 \\
\hline
\end{tabular}

Abbreviations: $N_{\mathrm{a}}$, number of alleles; $A_{\mathrm{r}}$, allelic richness; $H_{\mathrm{o}}$ and $H_{\mathrm{e}}$, observed and expected heterozygosity.

Value in bold are significant using different values of $\theta$.

while Z. v. vivipara had quick decay of relatedness such that it is near zero by $40 \mathrm{~km}$. Moreover, F1, the relatedness in the first distance class (that is, the pairs of individuals closest to each other) was near 0.15 for $Z v$. carniolica, while it was 0.08 for $Z$. v. vivipara. This fine-scale structure shows high relatedness in $Z v$. carniolica for near and moderate distances. This is consistent with the stronger clustering that appears in STRUCTURE.

\section{Niche differences}

In the fine-scale analysis (high resolution, limited to Northern Italy) niche overlap between the two lineages was limited (Schoener's $D=0.333$, Supplementary Figure S3d). The equivalency between the two niches was significantly lower than expected by chance $(P=0.002)$, while similarity tests showed that the two niches are not more similar than expected by chance (all $P>0.27$; Supplementary Figures S3e and f).

Density profiles showed significant differences for all the considered environmental variables (bootstrap test: $P<0.001$ for all variables considered). Specifically, the vivipara lineage was associated with environments significantly colder, drier and with lower primary productivity (Figure 5). Results remained consistent if the analysis was performed over the whole range of the two subspecies: the equivalency between the two niches was much lower than expected by chance (Schoener's $D=0.409, P=0.002$ ). Density profiles confirmed significant differences for all the considered environmental variables, except maximum summer temperature (Supplementary Figure S4).

\section{DISCUSSION}

Identification of species' boundaries is essential for describing and understanding species' natural history, for implementing conservation strategies, and for assessing and monitoring biodiversity. For instance, the most comprehensive and worldwide recognized list of threatened species (the Red List drafted by the International Union for Conservation of Nature) focuses mainly on species, although it also assesses subspecies or populations (IUCN, 2014). Effective conservation policies to counteract biodiversity loss will depend on accurate knowledge of extant biodiversity, including precise delimitation of species' boundaries and description of the ecological, genetic and natural history features of each species. In this context, the multidisciplinary approach defined 'integrative taxonomy' can be of particular assistance for recognizing conservation units. Combining
mtDNA analyses, population genetics and ecological niche analyses allowed us to identify species boundaries in a lacertid lizard inhabiting European Alps. Specifically we found: (a) that the two lineages display contrasting patterns of genetic structure; (b) no evidence of hybridization between the two lineages; (c) that the two lineages occupy different ecological niches; and (d) evidence of genetic fragmentation, decline and isolation for some $Z$. v. carniolica populations.

Before the discovery of the first oviparous population, ascribable to the genus Zootoca in Slovenia and Carinthia (Austria, Mayer et al., 2000), it was assumed that only one lineage of $Z$. vivipara inhabited the Alps - the widespread, viviparous and currently classified Z. $v$. vivipara. This cryptic differentiation in natural history was probably ignored because the two lineages ( $Z$. v. vivipara and $Z$. v. carniolica) cannot be unequivocally distinguished by morphology (Guillaume et al., 2006). However, the two lineages differ at a major phenotypic trait (reproductive mode), and experienced long independent evolutionary histories, accumulating genetic divergence in allopatry, before their secondary contact in the Alpine chain (Surget-Groba et al., 2006; Cornetti et al., 2014). We confirmed here with an ample data set that the two lineages, currently classified as 'subspecies', live in sympatry and without hybridization in the Alps, and exploit distinct niches.

The initial mitochondrial survey, necessary for lineage identification, not only confirmed the profound genetic divergence at mitochondrial level (Figure 2a) but also interestingly revealed that the oviparous $Z$. $v$. carniolica is about 10 times more variable at cytb than $Z$. v. vivipara. This finding explains different demographic patterns for the two lineages; while the oviparous lineage remained isolated in refugia in the southern margin of Alpine chain and Po Plain during glacial period (Surget-Groba et al., 2002), the viviparous one colonized the Alps only recently from East (from Balkans or southern Russia, Surget-Groba et al., 2001). We also found an west-east decrease in nucleotide diversity in Z. v. carniolica (Table 2), with many locations in Northern-Eastern Italy showing no variation at mitochondrial DNA. This pattern is consistent with a scenario where oviparous populations remained isolated in a northwestern Italian refugium and then colonized northern Italy, Austria and Slovenia. Populations persisting in glacial refugia are generally characterized by high levels of genetic diversity as a result of a longer demographic history, while populations that recently colonized new areas typically show low genetic variation (Hewitt, 2000). 
Lack of gene flow between populations due to habitat loss and modification can reduce effective population sizes and increase genetic drift in isolated populations. Although Z. v. carniolica displayed higher genetic variation than $Z$. v. vivipara at $\mathrm{mtDNA}$, we found signals of genetic fragmentation in two oviparous populations showing reciprocally private haplotypes (that is, Ampola and Rasun, with OS6 and OS2 haplotypes, respectively), likely as a result of habitat discontinuity. Our finding, also confirmed by microsatellites data (see below) are concordant with the hypothesis that a combination of climate warming (Fenner et al., 2007) and anthropization (for example, drainage of peatbogs, Semenzato et al., 1996) have been contributing to reduction in ground surface of wetlands and thus reducing and fragmenting suitable habitat for Z. vivipara in Northern (especially Northeastern) Italy.

Microsatellite data confirm the long, independent evolutionary histories of oviparous and viviparous lineages, and support the hypothesis that they likely started to diverge long ago (at least 2.6

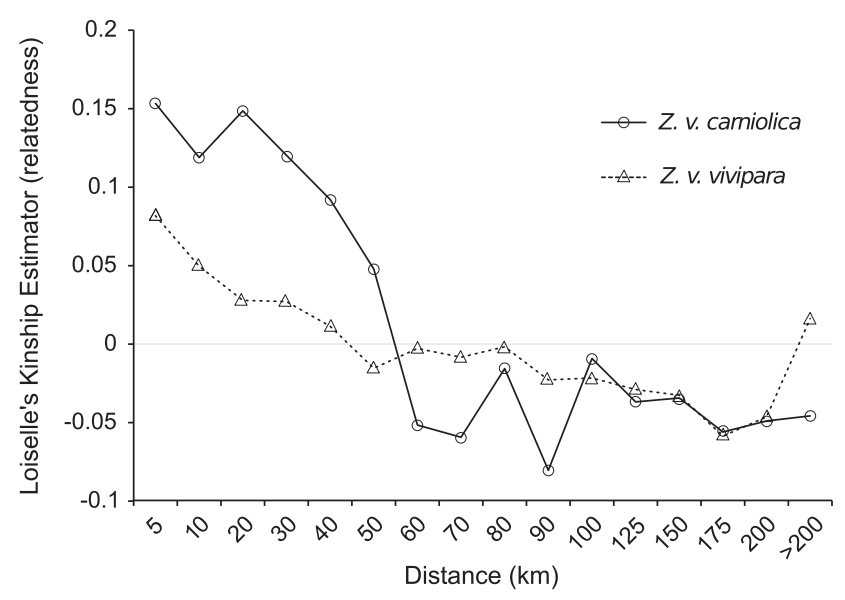

Figure 4 Spatial autocorrelograms for all pairs of individuals within distance classes, comparing Z. v. vivipara (dashed lines) and Z. v. carniolica (continuous lines).
Mya, according to Cornetti et al., 2014). Difficulties in correctly amplifying the same set of microsatellites, occurrence of private deletions in carniolica samples in the flanking region of two markers and high percentage of private alleles in each lineage supported the hypothesis of an ancient separation time between the two lineages (Surget-Groba et al., 2001; Cornetti et al., 2014). In addition, we could confidently exclude the presence of hybrid individuals in our sample set (Figure $2 b$ ), meaning that pre- or post-zygotic barriers likely arose in allopatry and promoted reproductive isolation after viviparal carniolica secondary contact. However, detailed investigations in the rare syntopic locations are needed to shed light on this aspect. Previous studies showed somehow contrasting results. For instance, Lindtke et al. (2010) hypothesized the occurrence of natural hybridization between $Z$. v. vivipara and Z. v. carniolica, on the basis of phenotypic traits, such as egg-retention time and eggshell thickness. Still, genetic data would be required to confirm the actual hybridization in Carinthia (Austria, Lindtke et al., 2010). Indeed, fine-scale analyses on a very-narrow contact zone found genetic reproductive isolation between the two lineages (Cornetti et al., 2015). It should also be remarked that gene flow exists between other mtDNA lineages of Z. vivipara. For instance, Mila et al., 2013 found asymmetric intregression among divergent mtDNA lineages of $Z$. v. louislantzi in the Pyrenees. However, the divergence time between such lineages was about 1 million years ago, much more recently than Z. v. vivipara and Z. v. carniolica.

The two lineages also showed contrasting patterns of genetic structure. In particular, while carniolica presented a well-defined genetic subdivision, vivipara did not, reflecting opposite demographic histories (Figure 3). The absence of clear genetic structure in Z. $v$. vivipara could reveal either extant gene flow or a recent and continuous expansion of the lineage across the Alps. The gene flow explanation is plausible since vivipara tends to live at higher altitudes than carniolica (Cornetti et al., 2014) and therefore it is likely less affected by the consequences of recent anthropogenic modifications and habitat discontinuity. Similarly, demographic processes such as recent colonization events and range expansion after last glacial maximum could explain the lack of genetic differentiation, since subpopulation divergence takes a long time to accumulate
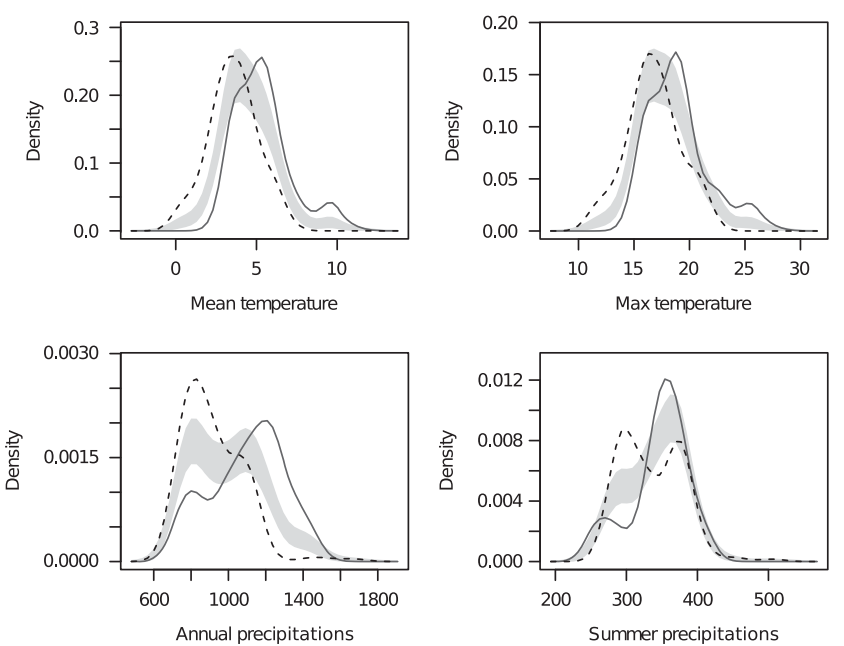
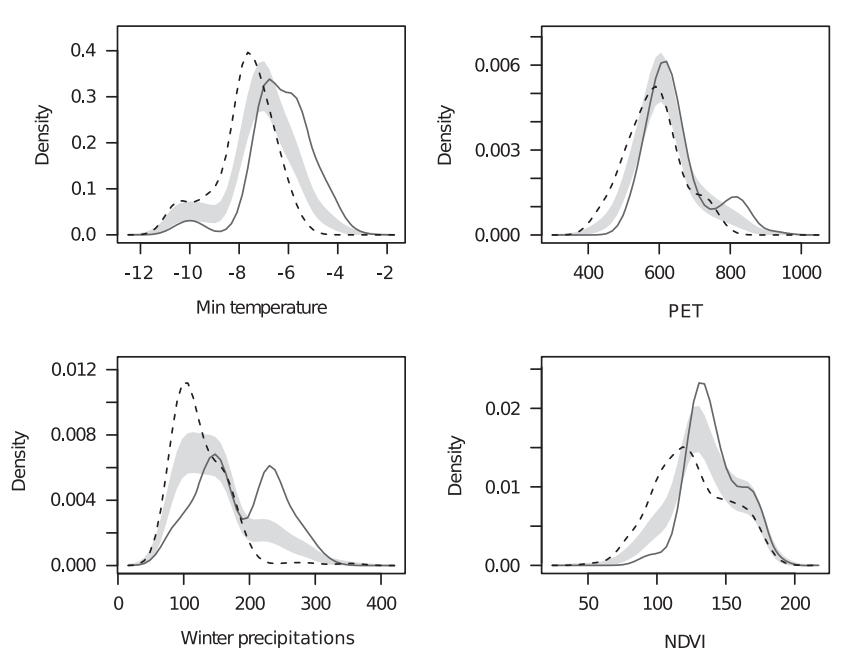

Figure 5 Density profiles comparing the frequency of Z. v. vivipara (dashed lines) and Z. $v$. carniolica (continuous lines) along the eight environmental variables: results of the fine-scale analysis. The gray areas represent $95 \%$ confidence interval obtained through bootstraps. If the density profile of one taxon is above the $95 \%$ confidence interval, is significantly associated to these environmental conditions; if it is below the $95 \%$ confidence interval, it is significantly less frequent than expected by chance. See Supplementary Figure S4 for the broad scale analysis. 
Table 2 MtDNA variation in Z. v. carniolica and Z. v. vivipara lineages

\begin{tabular}{lllll}
\hline Population & $N$ & $H$ & \multicolumn{1}{c}{$h$} & \multicolumn{1}{c}{$\pi$} \\
\hline $\begin{array}{l}\text { Z. v. carniolica } \\
\quad \text { Ampola }\end{array}$ & 17 & 1 & 0 & 0 \\
$\quad$ Eastern Alps & 32 & 2 & 0.1210 & 0.0005 \\
$\quad$ Piedmont & 29 & 5 & 0.7266 & 0.0052 \\
$\quad$ Lombardy & 22 & 3 & 0.4372 & 0.0017 \\
$\quad$ Rasun & 14 & 1 & 0 & 0 \\
& & & & \\
Z. v. vivipara & & & & 0.0012 \\
$\quad$ East of Brenner line & 96 & 4 & 0.4789 & 0.0009 \\
$\quad$ West of Brenner line & 94 & 4 & 0.3747 & \\
\hline Abbreviations: $H$, number of haplotypes; $h$, haplotype diversity; $\pi$, nucleotide diversity.
\end{tabular}

(Hewitt, 2000). It is also noteworthy that, while the mtDNA shows a much higher diversity for $Z$. v. carniolica, the microsatellite DNA variability shows an opposite pattern. This makes sense as the mtDNA shows the historical structure and the viviparous lineage probably colonized only recently the Alps, while microsatellites reflect current population structure and the recent fragmentation and bottlenecks in the oviparous lowland populations.

Only a longitudinal subdivision in two groups emerged for the $Z$. $v$. vivipara lineage, dividing populations from western and Eastern Alps, along the so-called 'Brenner line', (including the Adige Valley up to the Brenner Pass, Figure 1; Kerner, 1870). This was confirmed for $Z$. v. carniolica, although showing a stronger genetic structure (five well-defined groups, Figure 3a). When put in the broader context of the European Alps biogeography, the observed pattern of east-west differentiation across the Adige Valley confirms what was detected in some alpine animal and plant species. For instance, molecular data have ascertained genetic differentiation between western and eastern Adige Valley in the high-altitude butterfly Erebia euryale (Haubrick and Schmitt, 2007). Some authors (Penck and Bruckner, 1909; Van Husen, 1987) have suggested that two refugia of alpine plant species were present during the glacial oscillations in this region: one in southern Adamello west of the Adige Valley and the other in the Southwestern Dolomites east of the Adige. Indeed, there are plant species such as Phyteuma globulariifolium, an Alpine form of rampion, and the alpine speedwell, Veronica alpina, that can be genetically subdivided into two major groups, east and west of the Adige Valley (Schönswetter et al., 2002; Albach et al., 2006). More recently, the area between Lake Garda and Innsbruck, including the Adige Valley, has been invoked as a zone of genetic discontinuity delimiting Eastern and Western Alpine populations of many other species (Thiel-Egenter et al., 2009). Interestingly, the 'Brenner line', was first recognized as delimiting eastern and western distributions of plant species in the nineteenth century (Kerner, 1870). The outcome of our survey corroborates the hypothesis that the 'Brenner line' might act as an important barrier for historical recolonization and contemporary gene flow for many alpine species (for example, Crestanello et al., 2009; Thiel-Egenter et al., 2009) and suggests that it may be particularly important for species showing limited dispersal, such as small vertebrates.

Differences in isolation by distance patterns were also observed, with Z. v. carniolica displaying higher relatedness at short distances than $Z$. v. vivipara. These results are congruent with the evidence that anthropization and urban sprawl in lowlands/low-elevation valleys has a potential negative effect on biodiversity in the Alps (Nobis et al., 2009) as increasing occurrence of unsuitable habitats might prevent gene flow (for example, Trizio et al., 2005), and increase inbreeding. For instance, we identified two populations constituting two distinct groups: Ampola and Rasun. These populations are emblematic examples of isolated populations of conservation concern; they showed the lowest estimated number of alleles, allelic richness and heterozygosity (Table 1), and no genetic variation at mtDNA. These results likely reflect the consequences of genetic drift on small and isolated populations.

Conservation managers, in order to prioritize species/populations to be preserved, are usually concerned by changes in $N_{\mathrm{e}}$, especially recent demographic collapse, which may be revealed in genetic data. One method for detecting a recent reduction in population size (bottleneck) showed no significant reduction in the two lineages of $Z$. vivipara, while different demographic dynamics were revealed by a method for estimating bottleneck occurrence in a more distant past (M_P_VAL). Specifically, while no evidence of population reduction was highlighted for the viviparous lineage, four out of five clusters of Z. v. carniolica likely suffered from bottlenecks according to M-ratio results. This indication was confirmed by preliminary Approximate Bayesian Computation analyses (unpublished results), although different demographic dynamics could be biased by unbalanced population sizes.

Ecological differences between the two lineages are strong, and the differentiation was evident both in fine-scale and in range-wide analyses. Although Z. v. vivipara and Z. v. carionica are sympatric in the Alpine chain, cases of syntopy are extremely rare, and the two lineages show a niche overlap of only 0.33 , a value much lower than expected under equivalency expectations (Supplementary Figure S3). In practice, the two lineages show significant differences for nearly all the analyzed variables. The oviparous lineage tends to occupy more productive habitats, showing precipitation about 50\% higher than areas occupied by the viviparous populations (Figure 5). Furthermore, strong differences are detected for variables representing the thermal environment (in particular mean and minimum annual temperature). For example, while vivipara is associated to areas with mean annual temperature of about $3^{\circ} \mathrm{C}$, carniolica is more frequent in habitats where mean temperature is above $5^{\circ} \mathrm{C}$ (Figure 5, Supplementary Figure S4). Such strong differences for ecological niches are likely related to the different reproductive mode showed by the two lineages, and to the biological/ecological processes that determined the transition from oviparity to viviparity. In fact in reptiles the transition toward viviparity is consistently associated with colonization of harsh and cold climates (Pyron and Burbrink, 2014). For instance, $Z$. v. vivipara individuals exhibit a higher cold tolerance than oviparous ones (Voituron et al., 2004) and offspring that develop inside the body of the females for longer are more likely to survive freezing temperatures (Blackburn, 2005), allowing the colonization of high latitudes and altitudes. Differences in thermal tolerance likely determine the distinct altitudinal distribution of the two lineages in the Alps, with the viviparous living at higher altitude than oviparous one, although an overlap of their altitudinal distribution has been documented in the Alpine chain (Cornetti et al., 2014).

The two lineages of $Z$. vivipara have different evolutionary histories, contrasting local and regional genetic structure and well-separated ecological niches. These results and the lack of evidence of hybridization suggest that Z. v. vivipara and Z. v. carniolica should be considered two separate species and two distinct conservation units, with different management strategies tailored according to their genetic structure and habitat. Particular attention should be given to the oviparous lineage that inhabits low-altitude habitats, which is threatened by increasing impact of human activities in Po Plain 
and Southern Alps, especially since population decline and local extinctions have been already reported in this area (Sindaco et al., 2006). If the distinctiveness between the two lineages is overlooked, there is the risk of an unnoticed decline and extinction of the oviparous lineage. Meanwhile vivipara has a very broad geographic range (Supplementary Figure S1), mostly stable populations, and is considered a 'least concern' species (IUCN, 2014). Nevertheless, it has also been demonstrated that if evolution of viviparity favored successful colonization of cold climate for reptiles, viviparous lineages are, at the same time, associated with an increased risk of extinction due to rapid climate warming since they are likely to remain adaptively constrained to cold environments (Pincheira-Dinoso et al., 2013) posing, hence, a threat for Z. v. vivipara population persistence, as well. In sum, the application of integrative taxonomy allowed the correct identification of evolutionary significant units that should be managed differently with $Z$. $v$. carniolica managed to avoid extinction because of habitat loss, and Z. v. vivipara managed for the impact of climate change.

\section{DATA ARCHIVING}

Newly discovered cytb haplotypes are achieved in GenBank (KM522844 - KM522847). Microsatellite genotypes available from the Dryad Digital Repository: http://dx.doi.org/10.5061/dryad.dr4s8.

\section{CONFLICT OF INTEREST}

The authors declare no conflict of interest.

\section{ACKNOWLEDGEMENTS}

We thank Barbara Crestanello, Samuele Ghielmi, Giovanni Giovine and Alexis Marchesini for their help during sampling. Andrea Gandolfi is warmly thanked for his help in figure editing. This research was partially funded by Autonomous Province of Trento (Italy) as part of the ACE-SAP project (University and Scientific Research Service regulation number 23, 12 June 2008) and by FIRS $>$ T (FEM International Research School of Trentino). SH was funded as a Postdoctoral Fellow at the National Institute for Mathematical and Biological Synthesis (NIMBioS), an Institute sponsored by the National Science Foundation through NSF Award \#DBI-1300426, with additional support from the University of Tennessee, Knoxville.

Albach DC, Schönswetter P, Tribsch A (2006). Comparative phylogeography of the Veronica alpina complex in Europe and North America. Mol Ecol 15: 3269-3286.

Anderson EC, Thompson EA (2002). A model-based method for identifying species hybrids using multilocus genetic data. Genetics 160: 1217-1229.

Arribas OJ (2009). Morphological variability of the Cantabro-Pyrenean populations of Zootoca vivipara (JACQUIN, 1787) with description of a new subspecies. Herpetozoa 21: 123-146.

Bandelt HJ, Forster P, Röhl A (1999). Median-joining networks for inferring intraspecific phylogenies. Mol Biol Evol 16: 37-48

Belkhir K, Borsa P, Chikhi L, Raufaste N, Bonhomme F (2004). GENETIX 4.05, logicie sous Windows TM pour la génétique des populations. Laboratoire Génome, Populations, Interactions, CNRS UMR 5171, Université de Montpellier II, Montpellier (France).

Bickford D, Lohman DJ, Sohdi NS, Ng PKL, Meier R, Winker K et al. (2007). Cryptic species as a window on diversity and conservation. Trends Ecol Evol 22: 148-155.

Blackburn DG (2005). Amniote perspectives on the evolution of viviparity. In: Grier H, Uribe MC (eds). Viviparity in Fishes. Homestead, FL: New Life Publications, pp 319-340.

Boudjemadi K, Martin O, Simon JC, Estoup A (1999). Development and cross-species comparison of microsatellite markers in two lizard species, Lacerta vivipara and Podarcis muralis. Mol Ecol 8: 513-525.

Bourguignon T, Šobotník J, Hanus R, Krasulova J, Vrkoslac V, Cvacka J et al. (2013). Delineating species boundaries using an iterative taxonomic approach: The case of soldierless termites (Isoptera, Termitidae, Apicotermitinae). Mol Phyl Evol 69: 694-703.

Bowman AW, Azzalini A (2010). R package 'sm': nonparametric smoothing methods (version 6022.2-4). www.r-project.org
Broennimann O, Fitzpatrick MC, Pearman PB, Petitpierre B, Pellissier L, Yoccoz (2012). Measuring ecological niche overlap from occurrence and spatial environmental data. Global Ecol Biogeogr 21: 841-897.

Cornetti L, Menegon M, Giovine G, Heulin B, Vernesi C (2014). Mitochondrial and nuclear survey of Zootoca vivipara across the Eastern Italian Alps: evolutionary relationships, historical demography and conservation implications. PLoS One 9: e85912.

Cornetti L, Belluardo F, Ghielmi S, Giovine G, Ficetola GF, Bertorelle G et al. (2015). Reproductive isolation between oviparous and viviparous lineages of Zootoca vivipara in a contact zone. Biol J Linn Soc 114: 566-573.

Crestanello B, Pecchioli E, Vernesi C, Mona S, Martinkova N, Janiga M et al. (2009). The genetic impact of translocations and habitat fragmentation in chamois (Rupicapra) spp. $J$ Hered 100: 691-708.

Dayrat B (2005). Towards integrative taxonomy. Biol J Linn Soc 85: 407-415.

Dent EA, Von Holdt BM (2012). STRUCTURE HARVESTER: a website and program for visualizing STRUCTURE output and implementing the Evanno method. Conserv Genet Resour 4: 359-361.

Di Rienzo A, Peterson AC, Garza JC, Valdes AM, Slatkin M, Freimer NB (1994). Mutational processes of simple sequence repeat loci in human populations. P Natl Acad Sci Usa 91: 3166-3170.

Estoup A, Angers B (1998). Microsatellites and minisatellites for molecular ecology: theoretical and empirical considerations. In: Carvalho GR (eds). Advances in Molecular Ecology. (NATO Science Series A: Life Sciences - Vol. 306), IOS Press: Amsterdam, pp 55-86.

Evanno G, Regnaut S, Goudet J (2005). Detecting the number of clusters of individuals using the software structure: a simulation study. Mol Ecol 14: 2611-2620.

Excoffier L, Lischer HEL (2010). Arlequin suite ver 3.5: A new series of programs to perform population genetics analyses under Linux and Windows. Mol Ecol Resour 10: 564-567.

Fenner N, Ostle NJ, McNamara N, Sparks T, Harmens H, Reynolds B et al. (2007). Elevated $\mathrm{CO}_{2}$ effects on peatland plant community carbon dynamics and DOC production. Ecosystems 10: 635-647.

Garza JC, Williamson EG (2001). Detection of reduction in population size using data from microsatellite loci. Mol Ecol 10: 305-318.

Goudet J (1995). FSTAT Version 1.2: a computer program to calculate F-statistics. J Hered 86: 485-486.

Guillaume CP, Heulin B, Pavlinov IY, Semenov DV, Bea A, Vogrin N et al. (2006). Morphological variations in the common lizard, Lacerta (Zootoca) vivipara. Russ $J$ Herpetol 13: 1-10.

Hardy OJ, Vekemans X (2002). SPAGEDi: a versatile computer program to analyse spatial genetic structure at the individual or population levels. Mol Ecol Notes 2: 618-620.

Haubrich K, Schmitt T (2007). Cryptic differentiation in alpine-endemic, high-altitude butterflies reveals down-slope glacial refugia. Mol Ecol 16: 3643-3658.

Hewitt GM (2000). The genetic legacy of the Quaternary ice ages. Nature 405: 907-913.

Hijmans RJ, Cameron SE, Parra JL, Jones PG, Jarvis A (2005). High resolution interpolated climate surfaces for global land areas. Int J Climatol 25: 1965-1978.

IUCN (2014). The IUCN Red List of Threatened Species. Version 2014.3.

Kerner A (1870). Die natürlichen Floren im Gelände der Deutschen Alpen. Fromann, Jena.

Koh LP, Dunn RR, Sodhi NS, Colwell RK, Proctor HC, Smith VS (2004). Species coextinctions and the biodiversity crisis. Science 305: 1632-1634.

Lindtke D, Mayer W, Böhme W (2010). Identification of a contact zone between oviparous and viviparous common lizards (Zootoca vivipara) in central Europe: reproductive strategies and natural hybridization. Salamandra 46: 73-82.

Loiselle BA, Sork VL, Nason J, Graham C (1995). Spatial genetic structure of a tropical understory shrub, Psychotria officinalis (Rubiaceae). Am J Bot 82: 1420-1425.

Luikart G, Cornuet JM (1998). Empirical evaluation of a test for identifying recently bottlenecked populations from allele frequency data. Conserv Biol 12: 228-237.

Manni F, Guérard E, Heyer E (2004). Geographic patterns of (genetic, morphologic, linguistic) variation: how barriers can be detected by 'Monmonier's algorithm'. Hum Biol 76: 173-190.

Mayer W, Böhme W, Tiedemann F, Bischoff W (2000). On oviparous populations of Zootoca vivipara (Jacquin, 1787) in South-eastern Central Europe and their phylogenetic relationship to neighbouring viviparous and South-west Europe-an oviparous populations. Herpetozoa 13: 59-69.

Milá B, Surget-Groba Y, Heulin B, Gosá A, Fitze PS (2013). Multilocus phylogeography of the common lizard Zootoca vivipara at the Ibero-Pyrenean suture zone reveals lowland barriers and high-elevation introgression. BMC Evol Biol 13: 192

Nobis MP, Jaeger JAG, Zimmermann NE (2009). Neophyte species richness at the landscape scale under urban sprawl and climate warming. Divers Distrib 15: 928-939.

Peery MZ, Kirby R, Reid BN, Stoelting R, Doucet-Beer E, Robinson S et al. (2012). Reliability of genetic bottleneck tests for detecting recent population declines. Mol Ecol 21: 3403-3418

Penck A, Bruckner E (1909). Die Alpen im Eiszeitalter. Leipzig, Germany: Tauchnitz.

Pincheira-Dinoso D, Tregenza T, Witt MJ, Hodgson DJ (2013). The evolution of viviparity opens opportunities for lizard radiation but drives it into a climatic cul-de-sac. Glob Ecol Biogeogr 22: 857-867.

Piry SG, Luikart G, Cornuet JM (1999). BOTTLENECK: a computer program for detecting recent reductions in the effective population size using allele frequency data. J Hered 90: 502-503.

Pritchard JK, Stephens M, Donnelly P (2000). Inference of population structure using multilocus genotype data. Genetics 155: 945-959. 
Pyron RA, Burbrink FT (2014). Early origin of viviparity and multiple reversions to oviparity in squamate reptiles. Ecol Lett 17: 13-21.

Raxworthy CJ, Ingram CM, Rabibisoa N, Pearson RG (2007). Applications of ecological niche modeling for species delimitation: a review and empirical evaluation using day geckos (Phelsuma) from Madagascar. Syst Biol 56: 907-923.

Remon N, Vila M, Galán P, Naveira H (2008). Isolation and characterization of polymorphic microsatellite markers in Iberolacerta monticola, and cross-species amplification in Iberolacerta galani and Zootoca vivipara. Mol Ecol Resour 8: 1351-1353.

Rödder D, Shulte U, Toledo LF (2013). High environmental niche overlap between the fungus Batrachochytrium dendrobatidis and invasive bullfrogs (Lithobates catesbeianus) enhance the potential of disease transmission in the Americas. North-West J Zool 9: 178-184.

Rodriguez MA, Belmontes JA, Hawkins BA (2005). Energy, water and large-scale patterns of reptile and amphibian species richness in Europe. Acta Oecol 28: 65-70.

Schönswetter P, Tribsch A, Barfuss M, Niklfeld H (2002). Several Pleistocene refugia detected in the high alpine plant Phyteuma globulariifolium Sternb. \& Hoppe (Campanulaceaea) in the European Alps. Mol Ecol 11: 2637-2647.

Semenzato M, Richard J, Amato S (1996). Boschi e risorgive planiziali: ambienti importanti per il mantenimento della continuità distributiva del popolamento erpetologico tra l'area Montana e quella di pianura del Veneto. St Trent Sci Nat Acta Biol 79. 59-69.

Sindaco R, Doria G, Razzetti E, Bernini F (2006). Atlas of Italian amphibians and reptiles. Firenze: Societas Herpetologica Italica, Edizioni Polistampa.

Singhal S, Moritz C (2013). Reproductive isolation between phylogeographic lineages scales with divergence. P Roy Soc B 280: 2246.
Surget-Groba Y, Heulin B, Ghielmi S, Guillaume CP, Vogrin N (2002). Phylogeography and conservation of the populations of Zootoca vivipara carniolica. Biol Conserv 106: 365-372.

Surget-Groba Y, Heulin B, Guillaume CP, Puky M, Semenov D, Orlova V et al. (2006) Multiple origins of viviparity, or reversal from viviparity to oviparity? The European common lizard (Zootoca vivipara, Lacertidae) and the evolution of parity. Biol J Linn Soc 87: 1-11.

Surget-Groba Y, Heulin B, Guillaume CP, Thorpe R, Kupriyanova L (2001). Intraspecific phylogeography of Lacerta vivipara and the evolution of viviparity. Mol Phyl Evol 18 449-459.

Thiel-Egenter C, Holderegger R, Brodbeck S, IntraBioDiv-Consortium, Gugerli F (2009) Concordant genetic breaks, identified by combining clustering tessellation methods, in two co-distributed alpine plant species. Mol Ecol 18: 4495-4507.

Trizio I, Crestanello B, Galbusera P, Wauters LA, Tosi G, Matthysen E et al. (2005). Geographical distance and physical barriers shape the genetic structure of Eurasian red squirrels (Sciurus vulgaris) in the Italian Alps. Mol Ecol 14: 469-481.

Van Husen D (1987). Die Ostalpen in den Eiszeiten. Wien: Geologische Bundesanstalt. Van Oosterhout C, Hutchinson WF, Wills DPM, Shipley P (2004). Micro-Checker: software for identifying and correcting genotyping errors in microsatellite data. Mol Ecol Notes 4 535-538.

Voituron Y, Heulin B, Surget-Groba Y (2004). Comparison of the cold hardiness capacities of the oviparous and viviparous forms of Lacerta vivipara: a preliminary study. J Exp Zool Part A 301A: 367-373.

Warren DL, Glor RE, Turelli M (2008). Environmental niche equivalency versus conservatism: quantitative approaches to niche evolution. Evolution 62: 2868-2883.

Supplementary Information accompanies this paper on Heredity website (http://www.nature.com/hdy) 